\title{
Comparação da Qualidade do Bloqueio Oftálmico Periconal com Ropivacaína a 1\% e 0,75\% com Punção nos Pontos Infraorbitário Lateral e Medial da Órbita *
}

\author{
Comparison of Ophthalmic Periconal Blockade with $1 \%$ and \\ $0.75 \%$ Ropivacaine with Lateral Infraorbital and \\ Medial Canthus Punctures
}

Carlos Escobar Vásquez ${ }^{1}$, Marcelo Vechi Macuco, TSA ${ }^{2}$, Antonio Bedin, TSA ${ }^{2}$, Renato Almeida Couto de Castro, TSA ${ }^{3}$

\section{RESUMO}

Vásquez CE, Macuco MV, Bedin A, Castro RAC - Comparação da Qualidade do Bloqueio Oftálmico Periconal com Ropivacaína a $1 \%$ e $0,75 \%$ com Punção nos Pontos Infraorbitário Lateral e Medial da Órbita

Justificativa e Objetivos - São diversos os fármacos empregados e diversas as vias de abordagem da região peribulbar. Nosso objetivo foi investigar a qualidade do bloqueio oftálmico periconal, através de dois pontos de punção, utilizando ropivacaína em diferentes concentrações, sem adição de hialuronidase.

Método - Foram estudados 50 pacientes submetidos à cirurgia oftalmológica, distribuídos aleatoriamente em dois grupos: R1 (Ropivacaína a 1\%) e R0,75 (Ropivacaína a 0,75\%). O volume de anestésico local injetado foi ajustado conforme a tolerância e distensibilidade do espaço periconal no ponto infraorbitário e, se necessário, um volume adicional de até $3 \mathrm{ml}$ no compartimento periconal medial da órbita. Escores de motilidade foram atribuídos a cada músculo reto. Foi avaliado também o grau de bloqueio motor dos músculos elevador da pálpebra superior e orbicular ocular. Os dados foram avaliados nos seguintes momentos: $M 0=$ imediatamente antes de realizar o bloqueio; $M 5=5$ minutos após o bloqueio; $M 10=10$ minutos após o bloqueio; e M15 = 15 minutos após o bloqueio.

Resultados - Dois pacientes do grupo $R 1$ e oito pacientes no grupo R0,75 necessitaram complementação, sendo esta diferença significativa. Em $72 \%$ dos casos, em ambos os grupos, foram realizadas somente as duas punções iniciais. $O$ volume total de anestésico e o volume empregado na primeira punção foram respectivamente, no grupo $R 1: 9,72 \pm 2,38 \mathrm{ml} \mathrm{e}$ $6,96 \pm 0,97 \mathrm{ml}$ e no grupo $R 0,75: 12,64 \pm 5,10 \mathrm{ml}$ e 7,36 $\pm 1,07$ $\mathrm{ml}$, apresentando também diferença significativa. Houve diferença significativa dos escores de motricidade nos momentos $M 10(R 1=1,6 ; R 0,75=3,8)$ e $M 15(R 1=1,04 ; R 0,75$ $=2,8)$.

Conclusões - Concluímos que a ropivacaína a 1\% mostrou-se mais eficiente do que a ropivacaína a 0,75\% quando utilizadas

\footnotetext{
* Recebido do (Received from) Hospital Municipal São José, CET/SBA/MEC do Serviço de Anestesiologia de Joinville - SAJ, SC

1. $M E_{2}$ do CET/SBA/MEC - SAJ

2. Anestesiologista. Co-responsável pelo CET/SBA/MEC - SAJ

3. Anestesiologista. Responsável pelo CET/SBA/MEC - SAJ
}

Apresentado (Submitted) em 29 de janeiro de 2002

Aceito (Accepted) para publicação em 02 de maio de 2002

Correspondência para (Mail to):

Dr. Carlos Escobar Vásquez

Rua Armando Odebrecht 70/305 - Bairro Ribeirão Fresco

89020-400 Blumenau, SC

(c) Sociedade Brasileira de Anestesiologia, 2002

Revista Brasileira de Anestesiologia

Vol. 52, Nº 6, Novembro - Dezembro, 2002 sem hialuronidase para realização de bloqueios oftálmicos periconais pelas técnicas apresentadas. Houve uma tendência a um menor número de punções com a ropivacaína a 1\%, e um número significativamente menor de pacientes necessitou de complementação neste mesmo grupo.

UNITERMOS: ANESTÉSICOS, Local: ropivacaína; CIRURGIA, Oftalmológica; TÉCNICAS ANESTÉSICAS, Regional: bloqueio periconal

\section{SUMMARY}

Vásquez CE, Macuco MV, Bedin A, Castro RAC - Comparison of Ophthalmic Periconal Blockade with $1 \%$ and $0.75 \%$ Ropivacaine with Lateral Infraorbital and Medial Canthus Punctures

Background and Objectives - Several drugs and methods are used for peribulbar blockade. Our objective was to investigate the quality of ophthalmic periconal blockade by the double puncture technique, with different ropivacaine concentrations and without hyaluronidase.

Methods - Participated in this study fifty patients submitted to ophthalmologic surgery, who were randomly allocated in two groups: R1 (1\% Ropivacaine) and R0.75 (0.75\% Ropivacaine). Local anesthetic volume was adjusted according to the tolerance and elasticity of the periconal space at the infraorbital site and, when necessary, not more than $3 \mathrm{ml}$ were used at the medial canthus site. Motor function scores were attributed to each rectus muscle. Motor block of the levator palpebrae superioris and orbicularis muscles was also evaluated. Data were evaluated in the following moments: $M 0=$ immediately before blockade; $M 5=5$ minutes after blockade; $M 10=10 \mathrm{~min}$ utes after blockade; and M15 = 15 minutes after blockade.

Results - Two R1 patients and eight R0.75 patients needed complementation and this difference was significant. In $72 \%$ of cases only 2 initial punctures were performed in both groups. Total anesthetic volume and first puncture volume were, respectively, for R1: $9.72 \pm 2.38 \mathrm{ml}$ and $6.96 \pm 0.97 \mathrm{ml}$; and for R0.75: $12.64 \pm 5.10 \mathrm{ml}$ and $7.36 \pm 1.07 \mathrm{ml}$, also with significant differences. There were significant motor function score differences in moments $M 10(R 1=1.6 ; R 0.75=3.8)$ and M15 (R1 = 1.04; R0.75 = 2.8)

Conclusions - We concluded that $1 \%$ ropivacaine is more effective than $0.75 \%$ ropivacaine when no hyaluronidase is used to perform ophthalmic periconal blockade by the double puncture technique. There was a trend to less punctures with $1 \%$ ropivacaine and a significantly lower number of patients needed complementation in this same group.

KEY WORDS: ANESTHETICS, Local: ropivacaine; ANESTHETIC TECHNIQUES, Regional: periconal blockade; SURGERY, Ophthalmologic 


\section{INTRODUÇÃO}

A cirurgia de catarata (facectomia) é, sem dúvida, a cirurgia oftálmica mais realizada em regime ambulatorial. $\mathrm{O}$ bloqueio oftálmico peribulbar, nestes casos, está amplamente difundido. Dentre os anestésicos locais, a ropivacaína em diferentes concentrações tem mostrado melhor perfil para cirurgia oftalmológica.

Como a hialuronidase parece influenciar na melhora da qualidade do bloqueio e na diminuição da pressão intraocular ${ }^{1}$, muitos autores utilizam-na de rotina. Mesmo reconhecendo as vantagens da hialuronidase, não a utilizamos em nosso estudo para podermos analisar o comportamento do anestésico em sua forma pura.

São várias as técnicas descritas para o bloqueio peribulbar. Uma variação mais eficiente da peribulbar é a peribulbar posterior, mais adequadamente chamada de periconal, na qual a solução anestésica é depositada com uma agulha mais longa posteriormente ao globo ocular, fora do cone muscular ${ }^{2,3}$.

A finalidade deste estudo foi investigar a qualidade do bloqueio utilizando ropivacaína em diferentes concentrações, sem adição de hialuronidase, com punção periconal no ponto infraorbitário lateral (junção do terço lateral com os dois terços mediais), complementando, quando necessário, com a punção periconal medial da órbita (canto medial) ${ }^{4}$.

\section{MÉTODO}

Após aprovação pela Comissão de Ética e assinatura do consentimento informado, 50 pacientes escalados para cirurgias eletivas oftalmológicas em regime ambulatorial participaram deste estudo. Os pacientes de ambos os sexos, na faixa etária dos 27 aos 86 anos de idade, estado físico ASA I e II, foram divididos aleatoriamente em dois grupos de 25 cada: R1 (Ropivacaína a 1\%) e R0,75 (Ropivacaína a 0,75\%).

Após a venóclise com cateter $20 \mathrm{G}$ ou $22 \mathrm{G}$, os pacientes foram monitorizados com eletrocardiograma contínuo, oximetria de pulso e pressão arterial sistólica, diastólica e média por método automático não invasivo. Todos receberam oxigênio a 0,5 L. $\mathrm{min}^{-1}$ através de cateter nasal. Foi administrada como sedação prévia ao bloqueio uma solução contendo fentanil $\left(10 \mu \mathrm{g} \cdot \mathrm{ml}^{-1}\right)$ com midazolam $\left(0,8 \mathrm{mg} \mathrm{ml}^{-1}\right)$, no total de $2 \mathrm{ml}$.

O bloqueio peribulbar foi realizado pelo método de dupla punção. O primeiro ponto foi o infraorbitário lateral nas junções do terço lateral com os dois terços mediais na tentativa de abordar o espaço peribulbar posterior ou mais propriamente chamado de periconal. A punção foi realizada com agulha 6,7 ou 8 , metálica, de ponta romba, própria para bloqueio oftálmico. O volume de anestésico variou conforme a tolerância e a distensibilidade deste espaço. A segunda punção, quando necessária, foi realizada no canto medial da fenda palpebral, segundo a técnica do bloqueio periconal medial da órbita com agulha padrão $25 \times 0,7$ (22G). Não foi ultrapassado neste ponto o volume de $3 \mathrm{ml}$. A seguir foi coloca- do o peso de McIntyre (baroftalmo: bolsa com mercúrio pesando 550 gramas) durante 15 minutos.

Escores de motilidade foram atribuídos a cada músculo reto: 0 = acinesia completa; 1 = motilidade diminuída; 2 = motilidade normal. Foram avaliados também o grau de bloqueio dos músculos elevador da pálpebra superior e orbicular ocular. Segundo este critério a motilidade total normal do globo ocular e das pálpebras corresponde a 12 pontos. A avaliação do bloqueio motor foi realizada nos momentos $\mathrm{M0}=$ imediatamente antes de realizar o bloqueio; M5 = 5 minutos após o bloqueio; $M 10=10$ minutos após o bloqueio; e M15 = 15 minutos após o bloqueio. Escore total igual ou menor que 4 indicou bloqueio motor satisfatório. Após a última avaliação, caso o escore fosse superior a 4 , nova dose adicional de ropivacaína era administrada, conforme a técnica descrita, seguida de baroftalmo e nova avaliação funcional.

Para avaliação da qualidade do bloqueio, o cirurgião foi orientado a responder se o bloqueio estava excelente (tudo estava perfeito), bom (poderia ser melhor), regular (não estava bom mas poderia iniciar a operação) ou péssimo (necessitava de complementação).

A comparação estatística adotada foi a seguinte: para os dados demográficos foram utilizados o teste $t$ de Student e o teste Exato de Fisher; para a comparação dos atributos entre os grupos o teste Exato de Fisher, o teste Qui-quadrado e o teste $t$ de Student; e para a média da somatória da motricidade nos diferentes momentos, o teste $t$ de Student.

\section{RESULTADOS}

Os grupos foram homogêneos com relação aos dados demográficos (Tabela I). O procedimento mais realizado foi a facectomia. No grupo R1 ocorreram dois transplantes corneanos e no R0,75 uma cirurgia de glaucoma.

Tabela I - Dados Demográficos

\begin{tabular}{llcc}
\hline & & $\mathrm{R} 1$ & $\mathrm{R} 0,75$ \\
\hline Idade (anos) * & Média \pm DP & $68,2 \pm 15,03$ & $68,76 \pm 9,95$ \\
& Moda & 49 & 71 \\
& Limites & 27 a 86 & 43 a 85 \\
Peso $(\mathrm{kg})^{*}$ & Média \pm DP & $65,88 \pm 11,96$ & $66,36 \pm 13,22$ \\
& Moda & 60 & 63 \\
Sexo & Limites & 44 a 100 & 39 a 92 \\
& Masculino & 12 & 11 \\
ASA & Feminino & 13 & 14 \\
& I & 4 & 4 \\
Procedimentos & Facectomias & 23 & 21 \\
& Outros & 2 & 24 \\
\hline
\end{tabular}

* Valores expressos pela Média \pm DP 
A tabela II resume os resultados e compara os atributos dos dois grupos. Houve diferença significativa no número de pacientes que necessitaram de complementação com nova série de punções: dois pacientes no grupo R1 e oito pacientes no grupo R0,75. Em $72 \%$ dos casos, média dos 2 grupos, foram realizadas apenas as duas punções iniciais e 4 pacientes estudados exigiram apenas uma única punção (Tabela III e Figura 1). O volume total de anestésico e o volume empregado na primeira punção foram respectivamente no grupo $\mathrm{R} 19,72 \pm 2,38 \mathrm{ml} \mathrm{e} \mathrm{6,96 \pm 0,97} \mathrm{ml}$ e no grupo $\mathrm{R} 0,75$ $12,64 \pm 5,10 \mathrm{ml}$ e $7,36 \pm 1,07 \mathrm{ml}$, apresentando diferença significativa.

Tabela II - Comparação dos Atributos entre os Grupos

\begin{tabular}{|c|c|c|c|}
\hline & & $\mathrm{R} 1$ & $\mathrm{R} 0,75$ \\
\hline Complementação & & $2(8 \%)$ & $8(32 \%)$ * \\
\hline \multirow[t]{3}{*}{ Número de punções } & Única & $3(12 \%)$ & $1(4 \%)$ \\
\hline & Duas & $20(80 \%)$ & $16(64 \%)$ \\
\hline & $>$ Duas & $2(8 \%)$ & $8(32 \%)$ \\
\hline \multirow[t]{3}{*}{ Volume total $(\mathrm{ml})$} & Média $\pm \mathrm{DP}$ & $9,72 \pm 2,38$ & $12,64 \pm 5,10^{* *}$ \\
\hline & Moda & 9 & 9 \\
\hline & Limites & 6 a 16 & 6 a 28 \\
\hline \multirow[t]{3}{*}{ Volume $1^{\text {a }}$ punção (ml) } & Média $\pm \mathrm{DP}$ & $6,96 \pm 0,97$ & $7,36 \pm 1,07^{\text {** }}$ \\
\hline & Moda & 7 & 7 \\
\hline & Limites & 5 a 9 & 6 a 9 \\
\hline \multirow{3}{*}{ Volume canto medial ( $\mathrm{ml}$ ) } & Média $\pm \mathrm{DP}$ & $2,32 \pm 1,03$ & $2,68 \pm 0,69$ \\
\hline & Moda & 3 & 3 \\
\hline & Limites & 0 a 3 & 0 a 3 \\
\hline \multirow[t]{2}{*}{ Qualidade } & Excelente & $19(76 \%)$ & $16(64 \%)$ \\
\hline & Boa & $6(24 \%)$ & $9(36 \%)$ \\
\hline Quemose & & $6(24 \%)$ & $7(28 \%)$ \\
\hline Sensação de dor & & $2(8 \%)$ & $3(12 \%)$ \\
\hline
\end{tabular}

${ }^{*} p<0,05$ - teste Exato de Fisher; ${ }^{* *} p<0,05$ - teste $t$ de Student

Tabela III - Número de Punções por Grupo

\begin{tabular}{lccc}
\hline Punções & R1 & R0,75 & Total \\
\hline 1 & $3(12 \%)$ & $1(4 \%)$ & $4(8 \%)$ \\
2 & $20(80 \%)$ & $16(64 \%)$ & $36(72 \%)$ \\
3 & $2(8 \%)$ & $3(12 \%)$ & $5(10 \%)$ \\
4 & $0(0 \%)$ & $4(16 \%)$ & $4(8 \%)$ \\
5 & $0(0 \%)$ & $1(4 \%)$ & $1(2 \%)$ \\
\hline
\end{tabular}

Na média da somatória da motricidade nos diferentes momentos, houve diferença significativa nos momentos M10 ( $R 1=1,6 ; R 0,75=3,8)$ e M15 (R1 = 1,04; R0,75=2,8), conforme ilustrado na tabela IV. Esta comparação está esquematizada na figura 2.

Revista Brasileira de Anestesiologia

Vol. 52, № 6, Novembro - Dezembro, 2002

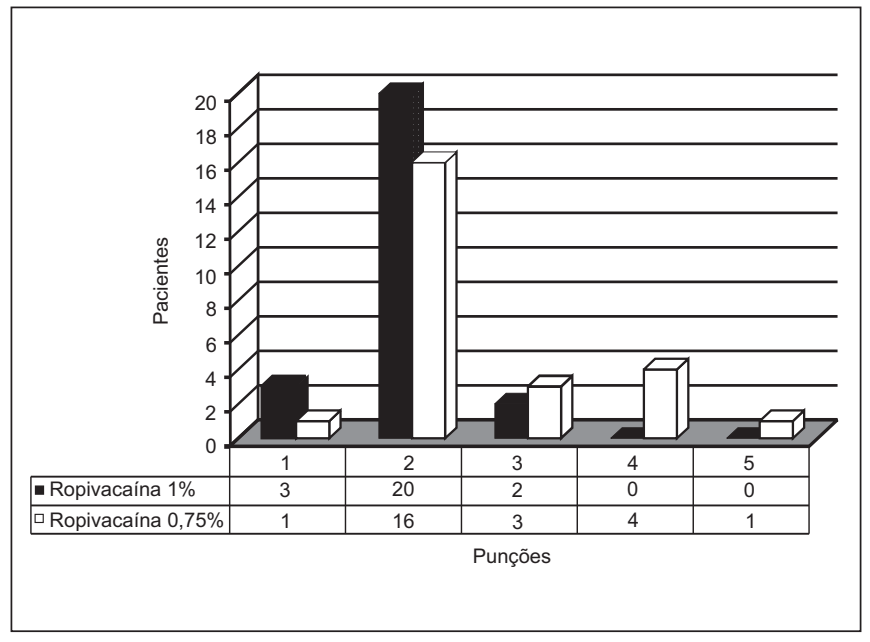

Figura 1 - Número de Punções por Grupo

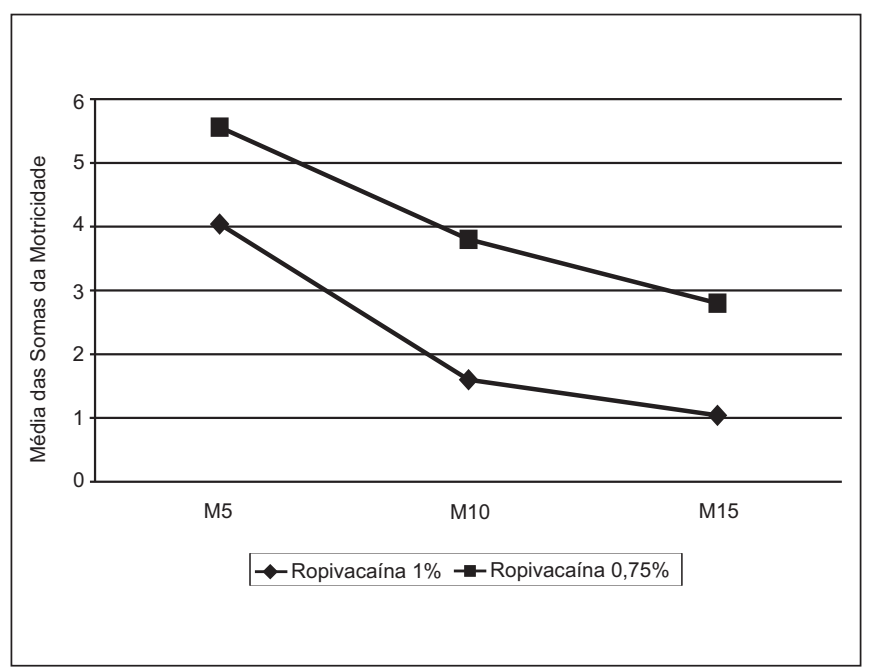

Figura 2 - Comparação da Somatória da Motricidade nos Diferentes Momentos

Tabela IV - Média das Somatórias da Motricidade nos Diferentes Momentos

\begin{tabular}{lcc}
\hline Momentos & $\mathrm{R} 1$ & $\mathrm{R} 0,75$ \\
\hline M5 & 4,04 & 5,56 \\
M10* & 1,6 & 3,8 \\
M15* & 1,04 & 2,8 \\
\hline
\end{tabular}

${ }^{*} p<0,05$ - teste $t$ de Student

\section{DISCUSSÃo}

Diversos trabalhos têm demonstrado que a ropivacaína é uma boa opção para anestesia peribulbar ${ }^{5,6}$. Na mesma concentração da bupivacaína, produz efeitos clinicamente indistingüíveis ${ }^{7}$. Promove ainda boa redução da pressão intraocular $^{8}$, efeito este bem evidente em relação à bupivacaína, provavelmente pelo seu efeito vasoconstritor intrínseco, ocasionando redução do volume sangüíneo na coróide ${ }^{9}$. 
A levobupivacaína e a bupivacaína racêmica promovem boas condições de anestesia cirúrgica ${ }^{10}$, teoricamente semelhante às da ropivacaína. Conforme outros autores, a alcalinização das soluções anestésicas melhoraria mais ainda a qualidade do bloqueio oftálmico ${ }^{11}$, por aumento da difusão. A hialuronidase facilita a difusão, mas também com isso diminui o tempo de ação do anestésico local, facilitando sua absorção ${ }^{12}$. Com a hialuronidase, os valores da pressão intraocular ficam menores e a qualidade do bloqueio motor aparentemente é melhor ${ }^{1,9}$

Diferentemente de outros autores, no nosso estudo optamos por orientar os pacientes a manter suas medicações antihipertensivas normalmente e a sedação feita antes do bloqueio mostrou-se eficaz na maioria dos pacientes em mantê-los calmos e cooperativos ${ }^{13-14}$.

A complicação mais temida durante a punção oftálmica é, sem dúvida, a perfuração do globo ocular, felizmente incomum. As mais comuns são a quemose, os hematomas, entre outras. As complicações são decorrentes na sua grande maioria do tipo de técnica empregada na dependência do local de punção.

Atualmente a técnica para bloqueio peribulbar segundo as publicações nacionais restringe-se basicamente à dupla punção nos pontos infraorbitário lateral (junção do terço lateral com os dois terços mediais) e supraorbitário medial. A punção periconal, igualmente às outras técnicas, tem mostrado bons resultados quanto à instalação rápida e qualidade do bloqueio. O ponto de acesso na região caruncular ou canto medial é uma boa alternativa para o bloqueio peribulbar, mesmo como punção única ${ }^{15-18}$. Ripart e col., utilizando punção única na região caruncular, denominaram a técnica como injeção episcleral ou sub-capsular de Tenon, realizada lateralmente à carúncula na região semilunar da conjuntiva

${ }^{19}$. No entanto, esta técnica está associada a um aumento excessivo da pressão intraocular e formação de quemose ${ }^{20}$. Segundo Hustead e col., a punção para bloqueio periconal medial da órbita (medialmente à carúncula) está associada a menores índices de sangramento e não apresenta o risco de lesão do músculo oblíquo superior ou seus anexos, como na punção supraorbitária medial ${ }^{4}$. Por isso optamos pelas punções combinadas periconais nos pontos infraorbitário lateral e medial da órbita (Figura 3).

A comparação de necessidade de complementação anestésica foi significativa entre os grupos. No grupo R1, somente dois pacientes ( $8 \%$ ) necessitaram de complementação, enquanto no grupo $\mathrm{R} 0,75$, foi necessária complementação em oito deles (32\%). Quanto ao número de punções, apesar de não ter sido estatisticamente significativo $(p>0,05)$, observamos uma tendência em realizar um menor número de punções complementares no grupo R1. O volume total de anestésico injetado no grupo R1 foi menor. Igualmente, o volume administrado no primeiro ponto de punção foi menor no grupo R1. No compartimento periconal medial da órbita (canto medial) não houve diferença entre os grupos quanto ao volume injetado, já que limitamos seu valor a $3 \mathrm{ml}$ para todos os pacientes.

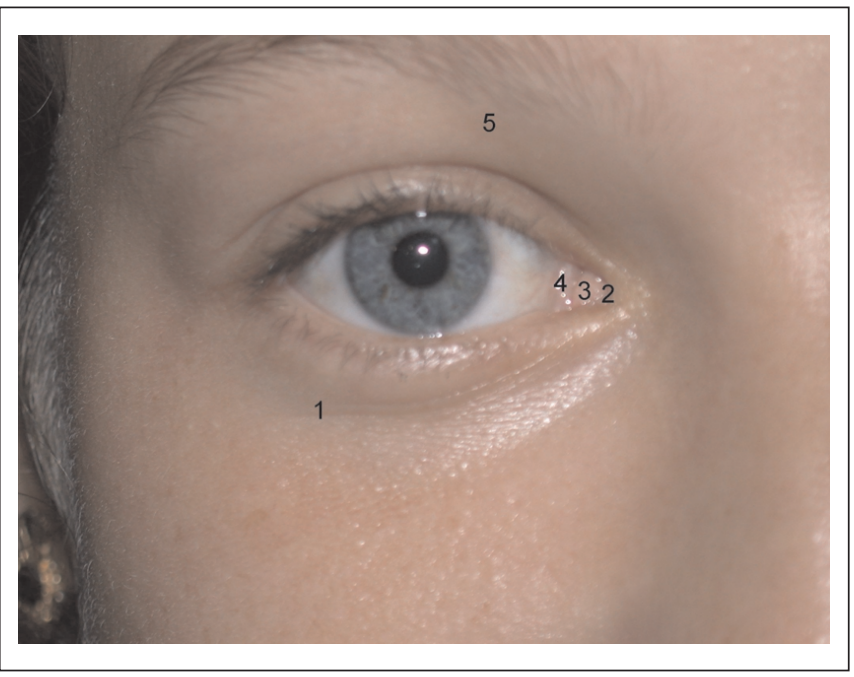

Figura 3 - Locais de Punção para Bloqueios Oftálmicos 1. Ponto infraorbitário lateral; 2. Ponto medial da órbita (canto medial): Técnica de Hustead; 3. Carúncula; 4. Região semilunar da conjuntiva: Técnica de Ripart; 5. Ponto supraorbitário media

A qualidade do bloqueio nos dois grupos foi boa ou excelente, não havendo diferença significativa entre eles. Nenhum grupo recebeu nota regular ou péssima.

Nos casos em que surgiu a quemose, sua importância clínica foi desprezível, segundo os oftalmologistas, não interferindo na realização do procedimento. Não observamos qualquer caso de hematoma ou outra intercorrência séria devida à punção. Dois pacientes do grupo $\mathrm{R} 1$ e três do $\mathrm{R} 0,75$ referiram sensação de fisgada ou dor no primeiro ponto passado pelo cirurgião para fixar o músculo reto superior, sem reclamar posteriormente da manipulação cirúrgica.

Asomatória total das notas de motricidade normal foi de doze (músculo reto lateral, músculo reto medial, músculo reto superior, músculo reto inferior, músculo orbicular das pálpebras e músculo elevador das pálpebras). Após anestesia, consideramos o valor quatro como sendo o mínimo para liberar a realização da cirurgia. Observamos assim que o grupo R1 foi mais eficiente na instalação do bloqueio.

Concluímos, através dos resultados apresentados neste estudo, que a ropivacaína a $1 \%$ mostrou-se mais eficiente do que a ropivacaína a $0,75 \%$, quando utilizadas sem hialuronidase para realização de bloqueios oftálmicos periconais pela técnica de dupla punção nos pontos infraorbitário lateral e medial da órbita (canto medial). A técnica apresentada é uma boa alternativa para bloqueios oftálmicos, mostrando-se eficiente, não produzindo intercorrências anestésicas importantes. Mesmo não se observando diferença significativa no número de punções, houve uma tendência a se realizar menos punções com ropivacaína a 1\%, e um número significativamente menor de pacientes necessitou de complementação neste grupo. 


\section{Comparison of Ophthalmic Periconal Blockade with $1 \%$ and $0.75 \%$ Ropivacaine with Lateral Infraorbital and Medial Can- thus Punctures}

Carlos Escobar Vásquez, M.D., Marcelo Vechi Macuco,TSA, M.D., Antonio Bedin, TSA, M.D., Renato Almeida Couto de Castro, TSA, M.D.

\section{INTRODUCTION}

Cataract surgery (facectomy) is clearly the most common outpatient ophthalmic surgery. Peribulbar ophthalmic block is widespread for such procedures. Among local anesthetics, different ropivacaine concentrations have shown a better profile for ophthalmologic surgeries.

Since hyaluronidase seems to influence blockade improvement and intraocular pressure decrease ${ }^{1}$, many authors use it as a routine. Even recognizing hyaluronidase's advantages, we have not used it in our study to be able to analyze the anesthetic drug behavior in its pure form.

There are several peribulbar techniques. A more efficient variation of the peribulbar block is the posterior peribulbar block, more adequately called periconal block, where the anesthetic solution is injected with a longer needle posteriorly to the eyeball, outside the muscular cone ${ }^{2,3}$.

This study aimed at investigating blockade quality with different ropivacaine concentrations (without hyaluronidase) with periconal puncture at the lateral infraorbitary site (junction of the lateral third with two medial thirds), complementing with medial orbital (medial canthus) periconal puncture, when needed $^{4}$.

\section{METHODS}

After the Ethical Committee approval and their informed consent, participated in this study 50 patients of both genders, aged 27 to 86 years, physical status ASA I and II, scheduled for elective outpatient ophthalmologic surgeries. Patients were randomly distributed in two groups of 25 : R1 $(1 \%$ ropivacaine) and $\mathrm{R} 0.75$ ( $0.75 \%$ ropivacaine).

After a venous access with a $20 \mathrm{G}$ or $22 \mathrm{G}$ catheter, patients were monitored with continuous ECG, pulse oximetry and non-invasive systolic, diastolic and mean blood pressure. All patients received $0.5 \mathrm{~L}$. $\mathrm{min}^{-1}$ oxygen through a nasal catheter. Patients were premedicated with a solution of fentanyl $\left(10 \mu \mathrm{g} \cdot \mathrm{ml}^{-1}\right)$ and midazolam $\left(0.8 \mathrm{mg} \cdot \mathrm{ml}^{-1}\right)$, in a total of $2 \mathrm{ml}$.

Peribulbar block was achieved by the double puncture method, being the first at the lateral infraorbital site at the junction of the lateral third with the two medial thirds, in an attempt to address the posterior peribulbar or, more exactly, the periconal space. Puncture was performed with 6,7 or 8 blunt tip metal needles, adequate for ophthalmic block. Anesthetic volume was adjusted according to the tolerance and elasticity of this space. The second puncture, when needed, was performed at the medial canthus, according to the medial orbital periconal blockade technique, with a standard 25 $x 0.7(22 \mathrm{G})$ needle. No more than $3 \mathrm{ml}$ were injected in this site. A Mcintyre weight was then applied (ocullopression: a mercury bag weighing $550 \mathrm{~g}$ ) and was left in place for 15 minutes.

Motor function scores were attributed to each rectus muscles: 0 = total akinesia; 1 = decreased motility; 2 = normal motility. Blockade levels of levator palpebrae superioris and orbicularis oculis muscles were also evaluated. According to these criteria, total normal motility of eyeball and eyelids corresponds to 12 points. Motor block was evaluated in the following moments: $\mathrm{M0}=$ immediately before blockade; $\mathrm{M} 5=5$ minutes after blockade; $M 10=10$ minutes after blockade and M15 $=15$ minutes after blockade. Total scores equal to or less than 4 would indicate a satisfactory blockade. After the last evaluation, and if the score would remain above 4 , an additional ropivacaine dose was administered according to the above described technique, followed by ocullopression and a new functional evaluation.

To evaluate blockade quality, the surgeon was asked to answer whether the blockade was excellent (everything perfect), good (could be better), average (was not good but surgery could be started) or poor (needing additional dose). Statistical comparison was as follows: Student's $t$ test and Fisher Exact test were used for demographics; Fisher Exact test, Chi-square test and Student's $t$ test were used for comparing attributes between groups; Student's $t$ test was used for total mean motility of different moments.

\section{RESULTS}

Groups were homogeneous in demographics data (Table I). Cataract was the most frequent surgical procedure. There were two corneal transplantations in R1 and one glaucoma surgery in R0.75.

Table I - Demographics Data

\begin{tabular}{llcc}
\hline & & R1 & R0.75 \\
\hline Age (years) * & Mean \pm SD & $68.2 \pm 15.03$ & $68.76 \pm 9.95$ \\
& Mode & 49 & 71 \\
Weight $(\mathrm{kg})^{*}$ & Limits & 27 a 86 & 43 a 85 \\
& Mean \pm SD & $65.88 \pm 11.96$ & $66.36 \pm 13.22$ \\
Gender & Limits & 60 & 63 \\
& Male & 44 a 100 & 39 a 92 \\
ASA & Female & 12 & 11 \\
& I & 13 & 14 \\
Procedures & II & 4 & 4 \\
& Facectomy & 23 & 21 \\
\hline
\end{tabular}

* Values expressed in Mean \pm SD 
Table II summarizes the results and compares the attributes for both groups. There has been a significant difference in the number of patients needing complementation with a new puncture: $2 \mathrm{R} 1$ patients and $8 \mathrm{R} 0.75$ patients. In $72 \%$ of cases (mean of both groups), only 2 initial punctures were performed and 4 patients required just one puncture (Table III and Figure 1). Total anesthetic volume and first puncture volume were, respectively, for R1: $9.72 \pm 2.38 \mathrm{ml}$ and $6.96 \pm 0.97$ $\mathrm{ml}$; and for R0.75: $12.64 \pm 5.10 \mathrm{ml}$ and $7.36 \pm 1.07 \mathrm{ml}$, with significant differences.

Table II - Comparison of Attributes between Groups

\begin{tabular}{|c|c|c|c|}
\hline & & $\mathrm{R} 1$ & $\mathrm{R} 0.75$ \\
\hline Complementation & & $2(8 \%)$ & $8(32 \%)$ * \\
\hline \multirow[t]{3}{*}{ Number of punctures } & Single & $3(12 \%)$ & $1(4 \%)$ \\
\hline & Two & $20(80 \%)$ & $16(64 \%)$ \\
\hline & $>$ Two & $2(8 \%)$ & $8(32 \%)$ \\
\hline \multirow[t]{3}{*}{ Total volume (ml) } & Mean \pm SD & $9.72 \pm 2.38$ & $12.64 \pm 5.10$ ** \\
\hline & Mode & 9 & 9 \\
\hline & Limits & 6 a 16 & 6 a 28 \\
\hline \multirow[t]{3}{*}{$1^{\text {st }}$ Puncture volume $(\mathrm{ml})$} & Mean \pm SD & $6.96 \pm 0.97$ & $7.36 \pm 1.07 * *$ \\
\hline & Mode & 7 & 7 \\
\hline & Limits & 5 a 9 & 6 a 9 \\
\hline \multirow[t]{3}{*}{ Medial canthus volume (ml) } & Mean \pm SD & $2.32 \pm 1.03$ & $2.68 \pm 0.69$ \\
\hline & Mode & 3 & 3 \\
\hline & Limits & 0 a 3 & 0 a 3 \\
\hline \multirow[t]{2}{*}{ Quality } & Excellent & $19(76 \%)$ & $16(64 \%)$ \\
\hline & Good & $6(24 \%)$ & $9(36 \%)$ \\
\hline Chemosis & & $6(24 \%)$ & $7(28 \%)$ \\
\hline Pain sensation & & $2(8 \%)$ & $3(12 \%)$ \\
\hline
\end{tabular}

${ }^{*} p<0.05$ - Fisher Exact test; ${ }^{* *} p<0.05$ - Student's $t$ test

Table III - Number of Punctures by Group

\begin{tabular}{lccc}
\hline Punctures & R1 & R0.75 & Total \\
\hline 1 & $3(12 \%)$ & $1(4 \%)$ & $4(8 \%)$ \\
2 & $20(80 \%)$ & $16(64 \%)$ & $36(72 \%)$ \\
3 & $2(8 \%)$ & $3(12 \%)$ & $5(10 \%)$ \\
4 & $0(0 \%)$ & $4(16 \%)$ & $4(8 \%)$ \\
5 & $0(0 \%)$ & $1(4 \%)$ & $1(2 \%)$ \\
\hline
\end{tabular}

Total mean motility in different moments showed significant differences in moments M10 (R1 = 1.6; R0.75 = 3.8) and M15 $(R 1=1.04 ; R 0.75=2.8)$, as shown in table IV and figure 2 .

Table IV - Total Motility Means In Different Moments

\begin{tabular}{lcc}
\hline Moments & $\mathrm{R} 1$ & $\mathrm{R} 0.75$ \\
\hline M5 & 4.04 & 5.56 \\
M10* & 1.6 & 3.8 \\
M15 $^{*}$ & 1.04 & 2.8 \\
\hline
\end{tabular}

${ }^{*} p<0.05$ - Student's $t$ test

686

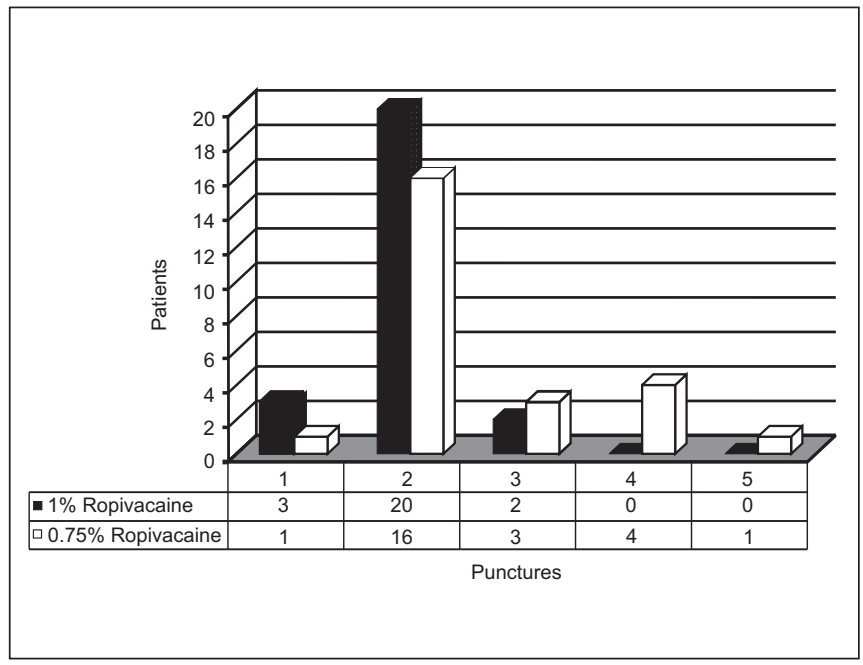

Figure 1 - Number of Punctures by Group

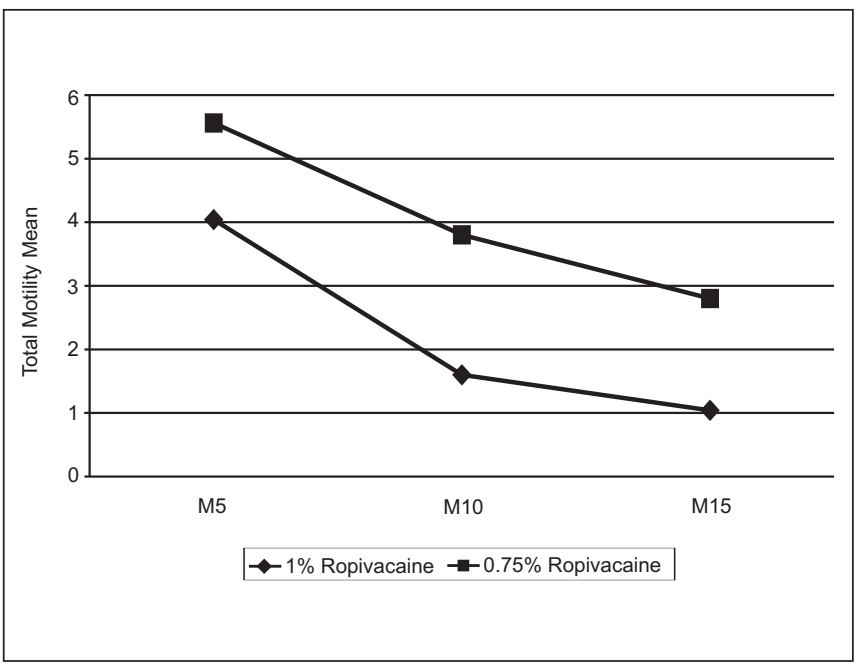

Figure 2 - Comparison of Total Motility in Different Moments

\section{DISCUSSION}

Several studies have shown that ropivacaine is a good option for peribulbar anesthesia ${ }^{5,6}$. In the same bupivacaine concentration, it produces similar effects ${ }^{7}$. It also promotes a good intraocular pressure decrease ${ }^{8}$, which is well apparent as compared to bupivacaine, probably due to its intrinsic vasoconstrictor effect, which decreases choroidal blood volume ${ }^{9}$.

Levobupivacaine and racemic bupivacaine promote good surgical anesthetic conditions ${ }^{10}$, in theory similar to those promoted by ropivacaine. According to other authors, the alkalization of anesthetic solutions would improve even more ophthalmic blockade quality ${ }^{11}$, by increasing diffusion.

Hyaluronidase helps diffusion, but also decreases local anesthetics action time, by helping their absorption ${ }^{12}$. With hyaluronidase, intraocular pressure values are lower and motor block quality is seemingly improved ${ }^{1,9}$.

Revista Brasileira de Anestesiologia Vol. 52, № 6 , Novembro - Dezembro, 2002 
Differently from other authors, we decided to advise patients to maintain their normal anti-hypertensive medication and pre-blockade sedation was effective in maintaining most patients relaxed and cooperative ${ }^{13,14}$.

The most feared complication during ophthalmic blockade is, undoubtedly, eyeball perforation, which fortunately is uncommon. Most common complications are ecchymosis and hematomas, among others. Complications are mostly a consequence of the technique, which is puncture site-dependent. Currently, the peribulbar technique is limited by national literature to double puncture in lateral infraorbital sites (junction of the lateral third with the two medial thirds) and medial supraorbital site. Periconal puncture, as other techniques, has shown to be effective in terms of fast onset and blockade quality. The access site at the medial canthus is a good peribulbar blockade alternative, even as a single puncture 15-18. Ripart et al., using a single medial canthus puncture, have named the technique episcleral or sub-Tenon injection, performed laterally to the caruncle at the semilunar region of the conjunctiva ${ }^{19}$. This technique, however, is associated to an excessive intraocular pressure increase and to the formation of ecchymosis ${ }^{20}$. According to Hustead et al., medial orbital periconal blockade (medially to the caruncle), is associated to less bleeding with no risk of injuring the obliquus superior muscle or its attachments, as it is the case with medial supraorbital puncture ${ }^{4}$. This is the reason why we have chosen combined periconal punctures at the lateral and medial infraorbital sites (Figure 3).

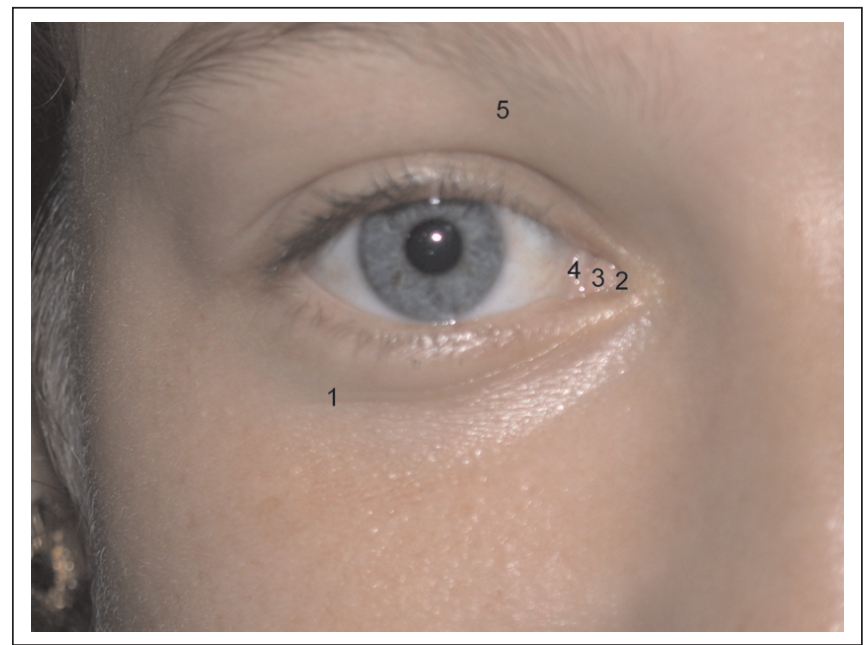

Figure 3 - Puncture Sites for Ophthalmologic Blockades 1. lateral infraorbital site; 2 medial orbital site (medial canthus): Hustead's technique; 3 . Caruncle; 4. Semilunar region of conjunctiva: Ripart's technique; 5 . Medial supraorbital site

The need for anesthetic complementation was significantly different between groups. Only two R1 patients ( $8 \%$ ) needed complementation as compared to eight $\mathrm{R} 0.75$ patients $(32 \%)$. Although not statistically significant $(p>0.05)$ there has been a trend for less additional punctures in R1. Total anesthetic volume was lower for R1. Similarly, the volume in- jected in the first puncture site was lower in R1. There have been no differences between groups in the volume injected in the medial canthus, since this volume was limited to $3 \mathrm{ml}$ for all patients.

Blockade quality for both groups varied from good to excellent without significant differences between them. No group was rated average or poor.

Chemosis had negligible clinical importance, according to ophthalmologists, and have not interfered with the procedure. There have been no hematomas or other severe puncture-related complications. Two R1 patients and three R0.75 patients referred pain or a sharp twinge at the first surgeon's stitch to fix the rectus superior muscle, without further complains.

Total normal motility score was 12 (rectus lateralis muscle, rectus medialis muscle, rectus superior muscle, rectus inferior muscle, orbicularis oculi muscle and levator palpebrae muscle). After anesthesia, four was the minimum score to start surgery and it was observed that blockade onset was more efficient in R1.

From the results obtained in this study, we concluded that $1 \%$ ropivacaine was more effective as compared to $0.75 \%$ ropivacaine, both without hyaluronidase, for periconal ophthalmic blocks using the double puncture technique in the lateral infraorbital and medial canthus sites. The technique is a good alternative for ophthalmic blocks and does not cause severe anesthetic complications. Even without significant differences in the number of punctures, there was a trend for less punctures with $1 \%$ ropivacaine and a significantly lower number of patients needed complementation in this group.

\section{REFERÊNCIAS - REFERENCES}

01. Serzedo PSM, Nociti JR, Zuccolotto EB et al - Bloqueio peribulbar com ropivacaína: influência da hialuronidase sobre a qualidade do bloqueio e a pressão intra-ocular. Rev Bras Anestesiol, 2001;51:202-207.

02. Vanetti LF - Anestesia para Oftalmologia, em: Yamashita AM, Takaoka F, Auler Jr JOC - Anestesiologia SAESP, $5^{a}$ Ed, São Paulo, Ateneu, 2001;811-830.

03. Ripart J, Lefant J, Coussaye JE et al - Peribulbar versus retrobulbar anesthesia for ophthalmic surgery: an anatomical comparison of extraconal and intraconal injections. Anesthesiology, 2001;94:56-62.

04. Hustead RF, Hamilton RC, Loken RG - Periocular local anesthesia: medial orbital as an alternative to superior nasal injection. J Cataract Refrac Surg, 1994;20:197-201.

05. Moraes SS, Leal Filho AV, Martins MP - Ropivacaína a 1\% em anestesia peribulbar para cirurgia de catarata. Rev Bras Anestesiol, 2000;50:248-250.

06. Nicholson G, Sutton B, Hall GM - Ropivacaine for peribulbar anaesthesia. Br J Anaesth, 1999;82:105-106.

07. Oliveira Filho GR, Nicolodi MA, Dal Mago AJ et al - Comparação entre a bupivacaína $0,75 \%$ e a ropivacaína $0,75 \%$ em bloqueio peribulbar. Rev Bras Anestesiol, 2000;50:217-220.

08. Serzedo PSM, Nociti JR, Zuccolotto EB et al - Pressão intraocular durante bloqueio peribulbar com ropivacaína a $1 \%$. Rev Bras Anestesiol, 2000;50:251-253. 
09. Serzedo PSM, Nociti JR, Zuccolotto EB et al - Pressão intraocular durante bloqueio peribulbar com ropivacaína ou bupivacaína: estudo comparativo. Rev Bras Anestesiol, 2000;50:341-344.

10. Mclure HA, Rubin AP - Comparison of $0.75 \%$ levobupivacaine with $0.75 \%$ racemic bupivacaine for peribulbar anaesthesia. Anaesthesia, 1998;53:1160-1164.

11. Prosser DP, Jones HM, Roberts JE - Peribulbar anaesthesia. Can J Anaesth, 1995;42:838-839

12. Cangiani LM - Anestesia em Oftalmologia, em: Manica J Anestesiologia: Princípios e Técnicas, $2^{\mathrm{a}} \mathrm{Ed}$, Porto Alegre, Artes Médicas, 1997;608-615.

13. Stocche RM, Garcia LV, Klamt JG - Medicação pré-anestésica com clonidina por via oral em cirurgia de catarata. Rev Bras Anestesiol, 2000;50:278-282.

14. Stocche RM, Klamt JG, Garcia LV - Clonidina venosa no controle da hipertensão arterial perioperatória em cirurgias de catarata. Estudo retrospectivo. Rev Bras Anestesiol, 2000;50:289-293.

15. Ripart J, Lefrant JY, Lalourcey L et al - Medial canthus (caruncle) single injection periocular anesthesia. Anesth Analg, 1996;83: 1234-1238.

16. Ripart J, Metge L, Prat-Pradal D et al - Medial canthus single-injection episcleral (sub-tenon anesthesia): computed tomography imaging. Anesth Analg, 1998;87:42-45.

17. Gillart T, Barrau P, Bazin JE et al - Lidocaine plus ropivacaine versus lidocaine plus bupivacaine for peribulbar anesthesia by single medial injection. Anesth Analg, 1999;89:1192-1201.

18. Ripart J, Lefrant J, L'Hermite J et al - Caruncle single injection episcleral (subtenon) anesthesia for cataract surgery: mepivacaine versus a lidocaine-bupivacaine mixture. Anesth Analg, 2000;91:107-109.

19. Ripart J, Benbabaali M, L'Hermite J et al - Ophthalmic blocks at the medial canthus. Anesthesiology, 2001;95:1533-1535.

20. Loken RG, Hamilton RC, Ripart J et al - Medial canthus (caruncle) single injection periocular anesthesia. Anesth Analg, 1997;85:707-708.

\section{RESUMEN}

Vásquez CE, Macuco MV, Bedin A, Castro RAC - Comparación de la Calidad del Bloqueo Oftálmico Periconal con Ropivacaína a $1 \%$ y $0,75 \%$ con Punción en los Puntos Infraorbitario Lateral y Medial de la Órbita

Justificativa y Objetivos - Diversos son los fármacos empleados y diversas las vías de abordaje de la región peribulbar. Nuestro objetivo fue investigar la calidad del bloqueo oftálmico periconal, a través de dos puntos de punción, utilizando ropivacaína en diferentes concentraciones, sin adición de hialuronidasa.

Método - Fueron estudiados 50 pacientes sometidos a cirugía oftalmológica, distribuidos aleatoriamente en dos grupos: R1 (Ropivacaína a 1\%) y R0,75 (Ropivacaína a 0,75\%). El volumen de anestésico local inyectado fue ajustado conforme la tolerancia y distensibilidad del espacio periconal en el punto infraorbitário y, si necesario, un volumen adicional de hasta 3 $\mathrm{ml}$ en el comportamiento periconal medial de la órbita. Contajes de motilidad fueron atribuidos a cada músculo recto. Fue evaluado también el grado de bloqueo motor de los músculos elevador de la pálpebra superior y orbicular ocular. Los datos fueron evaluados en los siguientes momentos: MO = inmediatamente antes de realizar el bloqueo; M5 = 5 minutos después del bloqueo; M10 = 10 minutos después del bloqueo; $y$ M15 = 15 minutos después del bloqueo.

Resultados - Dos pacientes del grupo $R 1$ y ocho pacientes en el grupo R0,75 necesitaron complementación siendo esta diferencia significativa. En $72 \%$ de los casos, en ambos grupos, fueron realizadas solamente las dos punciones iniciales. El volumen total de anestésico y el volumen empleado en la primera punción fueron respectivamente, en el grupo $R 1$ : $9,72 \pm 2,38 \mathrm{ml}$ y $6,96 \pm 0,97 \mathrm{ml}$ y en el grupo $R 0,75: 12,64 \pm 5,10$ ml y 7,36 $\pm 1,07 \mathrm{ml}$, presentando también diferencia significativa. Hubo diferencia significativa de los contajes de motricidad en los momentos $M 10(R 1=1,6 ; R 0,75=3,8)$ y $M 15$ $(R 1=1,04 ; R 0,75=2,8)$.

Conclusiones - Concluimos que la ropivacaína a 1\% se mostró más eficiente que la ropivacaína a $0,75 \%$ cuando utilizadas sin hialuronidasa para realización de bloqueos oftálmicos periconales por las técnicas presentadas. Hubo una tendencia a un número menor de punciones con la ropivacaína a 1\%, y un número significativamente menor de pacientes necesitó de complementación en este mismo grupo. 